\title{
The near-Sun streamer belt solar wind: turbulence and solar wind acceleration
}

\author{
C. H. K. Chen ${ }^{1}$, B. D. G. Chandran ${ }^{2}$, L. D. Woodham ${ }^{3}$, S. I. Jones ${ }^{4}$, J. C. Perez ${ }^{5}$, S. Bourouaine ${ }^{5,6}$, T. A. Bowen ${ }^{7}$, \\ K. G. Klein ${ }^{8}$, M. Moncuquet ${ }^{9}$, J. C. Kasper ${ }^{10,11}$, and S. D. Bale ${ }^{1,3,7,12}$ \\ ${ }^{1}$ School of Physics and Astronomy, Queen Mary University of London, London E1 4NS, UK \\ e-mail: christopher . chen@qmul . ac . uk \\ 2 Department of Physics and Astronomy, University of New Hampshire, Durham, NH 03824, USA \\ ${ }^{3}$ Department of Physics, Imperial College London, London SW7 2AZ, UK \\ ${ }^{4}$ NASA Goddard Space Flight Center, Greenbelt, MD 20771, USA \\ ${ }^{5}$ Department of Aerospace, Physics and Space Sciences, Florida Institute of Technology, Melbourne, FL 32901, USA \\ ${ }^{6}$ Johns Hopkins University Applied Physics Laboratory, Laurel, MD 20723, USA \\ ${ }^{7}$ Space Sciences Laboratory, University of California, Berkeley, CA 94720, USA \\ ${ }^{8}$ Lunar and Planetary Laboratory, University of Arizona, Tucson, AZ 85719, USA \\ ${ }^{9}$ LESIA, Observatoire de Paris, Université PSL, CNRS, Sorbonne Université, Université de Paris, 92195 Meudon, France \\ ${ }^{10}$ BWX Technologies, Inc., Washington, DC 20002, USA \\ ${ }^{11}$ Climate and Space Sciences and Engineering, University of Michigan, Ann Arbor, MI 48109, USA \\ ${ }^{12}$ Physics Department, University of California, Berkeley, CA 94720, USA
}

Received 6 November 2020 / Accepted 4 January 2021

\begin{abstract}
The fourth orbit of Parker Solar Probe (PSP) reached heliocentric distances down to $27.9 R_{\odot}$, allowing solar wind turbulence and acceleration mechanisms to be studied in situ closer to the Sun than previously possible. The turbulence properties were found to be significantly different in the inbound and outbound portions of PSP's fourth solar encounter, which was likely due to the proximity to the heliospheric current sheet (HCS) in the outbound period. Near the HCS, in the streamer belt wind, the turbulence was found to have lower amplitudes, higher magnetic compressibility, a steeper magnetic field spectrum (with a spectral index close to $-5 / 3$ rather than $-3 / 2$ ), a lower Alfvénicity, and a ' $1 / f$ ' break at much lower frequencies. These are also features of slow wind at 1 au, suggesting the near-Sun streamer belt wind to be the prototypical slow solar wind. The transition in properties occurs at a predicted angular distance of $\approx 4^{\circ}$ from the HCS, suggesting $\approx 8^{\circ}$ as the full-width of the streamer belt wind at these distances. While the majority of the Alfvénic turbulence energy fluxes measured by PSP are consistent with those required for reflection-driven turbulence models of solar wind acceleration, the fluxes in the streamer belt are significantly lower than the model predictions, suggesting that additional mechanisms are necessary to explain the acceleration of the streamer belt solar wind.
\end{abstract}

Key words. solar wind - Sun: heliosphere - plasmas - turbulence - waves

\section{Introduction}

One of the major open questions in heliophysics is how the solar wind is accelerated to the high speeds measured in situ by spacecraft in the Solar System (Fox et al. 2016). Early models of solar wind generation, based on the pioneering work of Parker (1958), were able to reproduce the qualitative properties of the solar wind, although they could not explain all of the measured quantities seen at 1 au (see, e.g. the reviews of Parker 1965; Leer et al. 1982; Barnes 1992; Hollweg 2008; Hansteen \& Velli 2012; Cranmer et al. 2015, 2017). Turbulence is now thought to be one of the key processes playing a role in solar wind acceleration, providing both a source of energy to heat the corona (Coleman 1968) and a wave pressure to directly accelerate the wind (Alazraki \& Couturier 1971; Belcher 1971). Possible mechanisms for the driving of the turbulence include reflection of the outward-propagating Alfvén waves by the large-scale gradients (Heinemann \& Olbert 1980; Velli 1993) and velocity shears (Coleman 1968; Roberts et al. 1992). Models that incorporate these effects are now able to reproduce most solar wind conditions at $1 \mathrm{au}$ (e.g. Cranmer et al. 2007; Verdini et al. 2010; Chandran et al. 2011; van der Holst et al. 2014; Usmanov et al. 2018; Shoda et al. 2019), but more stringent tests come from comparing their predictions to measurements close to the Sun.

The nature of the turbulence in the solar wind and plasma turbulence in general is also a major open question (Bruno \& Carbone 2013; Alexandrova et al. 2013; Kiyani et al. 2015; Chen 2016). Initial results from Parker Solar Probe (PSP) have revealed many similarities, but also some key differences in the nearSun solar wind turbulence. Both the power levels and cascade rates were found to be several orders or magnitude larger at $\sim 36 R_{\odot}$ compared to 1 au (Bale et al. 2019; Chen et al. 2020; Bandyopadhyay et al. 2020). The turbulence was also found to be less magnetically compressible and more imbalanced, with a shallower magnetic field spectral index of $\approx-3 / 2$. The low compressibility and polarisation is consistent with a reduced slow mode component to the turbulence (Chen et al. 2020; Chaston et al. 2020). Both the outer scale and ion scale spectral breaks 
move to larger scales approximately linearly with heliocentric distance (Chen et al. 2020; Duan et al. 2020), indicating that the width of the magnetohydrodynamic (MHD) inertial range stays approximately constant over this distance range. The steep ionscale transition range, however, is more prominent closer to the Sun, indicating stronger dissipation or an increase in the cascade rate (Bowen et al. 2020), or perhaps a build-up of energy at these scales (Meyrand et al. 2020). The overall increase in the turbulence energy flux, compared to the bulk solar wind kinetic energy flux, was found by Chen et al. (2020) to be consistent with the reflection-driven turbulence solar wind model of Chandran et al. (2011), showing that this remains a viable mechanism to explain the acceleration of the open field wind. Other comparisons of the early PSP data to turbulence-driven models also report agreement (Bandyopadhyay et al. 2020; Réville et al. 2020a; Adhikari et al. 2020).

Much of the solar wind measured in the early PSP solar encounters has been of open-field coronal hole origin (Bale et al. 2019; Panasenco et al. 2020; Badman et al. 2020, 2021), although short periods of streamer belt wind near the heliospheric current sheet (HCS) were also identified (Szabo et al. 2020; Rouillard et al. 2020; Lavraud et al. 2020). Encounter 4, however, was different in that for the majority of the outbound portion, PSP was consistently in streamer belt plasma. In this Letter, the properties of turbulence during this encounter are presented. These are compared to the distance to the HCS to show the differences between the streamer belt wind and the open field wind. The turbulence energy flux is also compared to predictions of reflection-driven turbulence solar wind models to investigate the acceleration mechanisms of the streamer belt wind.

\section{Data}

Data from PSP (Fox et al. 2016), primarily from its fourth solar encounter, but also from all of the first four orbits, were used for this study. The magnetic field, $\mathbf{B}$, and electron density, $n_{\mathrm{e}}$, were obtained from the MAG and RFS/LFR instruments of the FIELDS suite (Bale et al. 2016); the electron density measurement is described in Moncuquet et al. (2020). The ion (proton) velocity, $\mathbf{v}_{\mathrm{i}}$, and temperature, $T_{\mathrm{i}}$ were primarily obtained from the SPAN-I (Livi et al. 2021), but also the SPC (Case et al. 2020), instruments of the SWEAP suite (Kasper et al. 2016). The SPANI data consist of bi-Maxwellian fits to the proton core population, described in Woodham et al. (2021), with the same selection criteria used for excluding bad fits from the dataset, and these data are used for $\mathbf{v}_{\mathrm{i}}$ and $T_{\mathrm{i}}$ unless stated otherwise. Since the fluctuations investigated in this Letter are at MHD scales, the solar wind velocity $\mathbf{v}$ is considered to be equal to $\mathbf{v}_{\mathbf{i}}$.

A time series of the data for Encounter 4 is shown in Fig. 1. Additional quantities plotted include the distance-normalised mass flux, $4 \pi \rho v_{\mathrm{r}} r^{2}$, where $\rho$ is the total mass density estimated as $\rho=m_{\mathrm{p}} n_{\mathrm{e}}\left(1+3 f_{\alpha}\right) /\left(1+f_{\alpha}\right)$, where $f_{\alpha}=0.05$ is the assumed alpha fraction of the ion number density, $v_{\mathrm{r}}$ is the radial solar wind speed, and $r$ is the radial distance of the spacecraft to the Sun, the distance-normalised kinetic energy flux, $2 \pi \rho v_{\mathrm{r}}^{3} r^{2}$, and the ion plasma beta, $\beta_{\mathrm{i}}=2 \mu_{0} n_{\mathrm{i}} k_{\mathrm{B}} T_{\mathrm{i}} / B^{2}$. Furthermore, $\phi_{\mathrm{HCS}}$ is the angle (centred at the Sun) of the spacecraft to the HCS estimated using the Wang-Sheeley-Arge (WSA) model, which consists of a potential-field source-surface (PFSS) model for the inner corona encased in a Schatten current sheet shell (Arge \& Pizzo 2000; Arge et al. 2003, 2004; Szabo et al. 2020). A zero-point corrected GONG synoptic magnetogram was chosen for the model's inner boundary condition, which was found to produce solar wind predictions that correspond well with the magnetic field

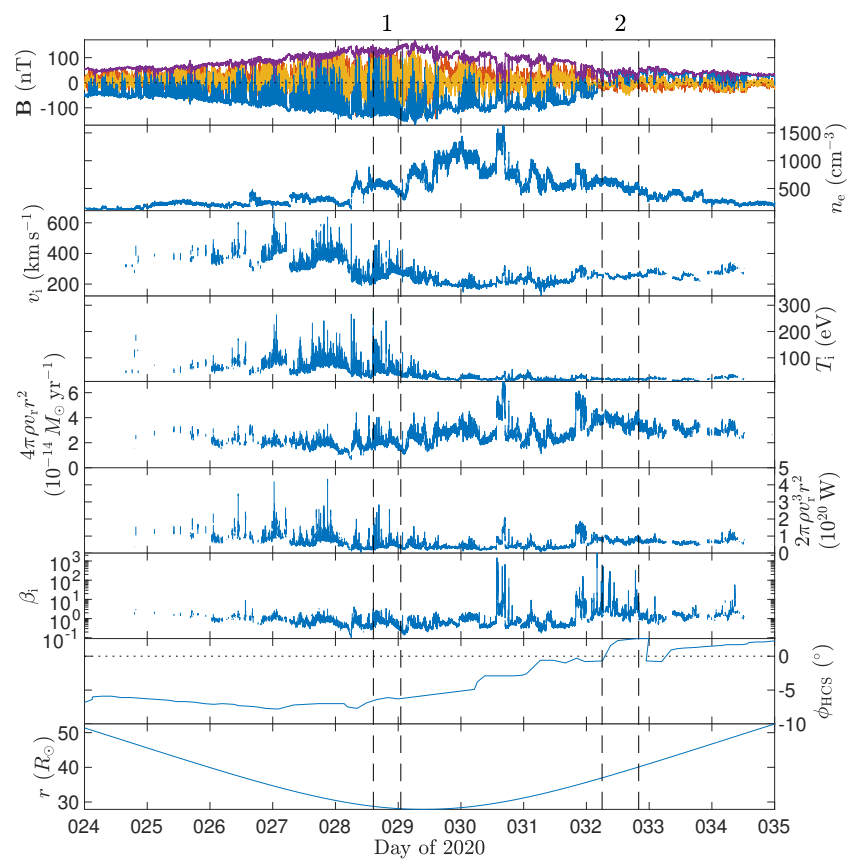

Fig. 1. Time series of Encounter 4 solar wind parameters: Magnetic field $\mathbf{B}$ in RTN coordinates (blue=radial, red=tangential, yellow $=$ normal, purple=magnitude), electron density $n_{\mathrm{e}}$, solar wind speed $v_{\mathrm{i}}$, ion temperature $T_{\mathrm{i}}$, mass flux $4 \pi \rho v_{\mathrm{r}} r^{2}$, energy flux $2 \pi \rho v_{\mathrm{r}}^{3} r^{2}$, ion beta $\beta_{\mathrm{i}}$, predicted angular distance to the heliospheric current sheet $\phi_{\mathrm{HCS}}$, and radial distance $r$. The vertical dashed lines indicate the intervals used to calculate the spectra in Fig. 6.

polarity inversion observed by PSP near the end of January. It can be seen that, unlike the previous encounters, the inbound and outbound portions had different solar wind properties: The outbound period had a higher density, lower speed and temperature, with a higher mass flux and plasma beta. These differences can be accounted for by the fact that PSP spent most of the outbound period close to the HCS.

\section{Results}

\subsection{Turbulence properties}

The 11-day period of Encounter 4 (days 24-34 of 2020) was divided into intervals of $300 \mathrm{~s}$ duration, roughly comparable to the outer scale (Chen et al. 2020; Parashar et al. 2020; Bandyopadhyay et al. 2020; Bourouaine et al. 2020), and in each a set of turbulence properties was calculated: the total rms magnetic fluctuation amplitude

$\delta B_{\mathrm{rms}}=\sqrt{\left\langle|\delta \mathbf{B}|^{2}\right\rangle}$,

where $\delta \mathbf{B}=\mathbf{B}-\mathbf{B}_{0}, \mathbf{B}_{0}=\langle\mathbf{B}\rangle$, and the angular brackets denote a time average; in this case, over each $300 \mathrm{~s}$ interval, the normalised rms fluctuation amplitude $\delta B / B_{0}$, the magnetic compressibility,

$C_{B}=\sqrt{\frac{\left\langle(\delta|\mathbf{B}|)^{2}\right\rangle}{\left\langle|\delta \mathbf{B}|^{2}\right\rangle}}$,

the normalised cross helicity,

$\sigma_{\mathrm{c}}=\frac{2\langle\delta \mathbf{v} \cdot \delta \mathbf{b}\rangle}{\left\langle|\delta \mathbf{v}|^{2}+|\delta \mathbf{b}|^{2}\right\rangle}$, 


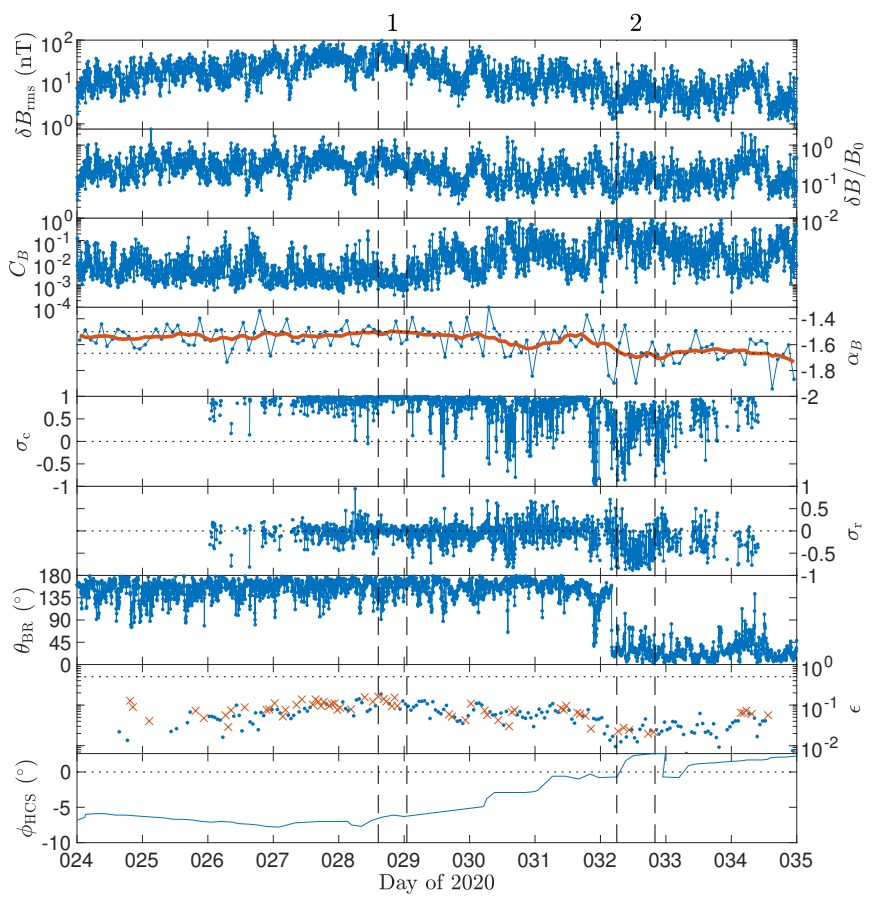

Fig. 2. Time series of Encounter 4 turbulence properties: rms magnetic fluctuation $\delta B_{\mathrm{rms}}$, normalised rms fluctuation $\delta B / B_{0}$, magnetic compressibility $C_{B}$, magnetic spectral index $\alpha_{B}$, cross helicity $\sigma_{\mathrm{c}}$, residual energy $\sigma_{\mathrm{r}}$, magnetic field angle to the radial $\theta_{\mathrm{BR}}$, Taylor hypothesis parameter $\epsilon$, and predicted angular distance to the heliospheric current sheet $\phi_{\mathrm{HCS}}$. The red line is a ten-point running mean of $\alpha_{B}$. Values of $\epsilon$ for which the Bourouaine \& Perez (2019) model is valid are marked with blue dots and points for which it is not valid are marked as red crosses. The vertical dashed lines indicate the intervals used to calculate the spectra in Fig. 6.

where $\mathbf{b}=\mathbf{B} / \sqrt{\mu_{0} \rho_{0}}$ and $\delta \mathbf{v}=\mathbf{v}-\langle\mathbf{v}\rangle$, the normalised residual energy,

$\sigma_{\mathrm{r}}=\frac{2\left\langle\delta \mathbf{z}^{+} \cdot \delta \mathbf{z}^{-}\right\rangle}{\left\langle\left|\delta \mathbf{z}^{+}\right|^{2}+\left|\delta \mathbf{z}^{-}\right|^{2}\right\rangle}$,

where the Elsasser fields are $\delta \mathbf{z}^{ \pm}=\delta \mathbf{v} \pm \delta \mathbf{b}$, and the angle between the magnetic field and the radial direction,

$\theta_{\mathrm{BR}}=\cos ^{-1}\left(\hat{\mathbf{B}}_{0} \cdot \hat{\mathbf{r}}\right)$

In the definition of $\mathbf{b}$, its sign is reversed if $\theta_{\mathrm{BR}}<90^{\circ}$ so that positive $\sigma_{\mathrm{c}}$ corresponds to Alfvénic propagation away from the Sun. In addition, the MHD inertial range magnetic field spectral index, $\alpha_{B}$, was calculated from the FFT of 2-hour intervals and fitting a power-law function in the range of spacecraft-frame frequencies $10^{-2} \mathrm{~Hz}<f_{\text {sc }}<10^{-1} \mathrm{~Hz}$.

A time series of these properties, along with the angular distance to the HCS, $\phi_{\mathrm{HCS}}$, is shown in Fig. 2. As expected, there is significant variability in all quantities, but there are also consistent trends over the encounter. The outbound portion appears to have lower fluctuation amplitudes, higher magnetic compressibility, and a steeper spectral index as well as to be less dominated by pure outward Alfvénic fluctuations $\left(\sigma_{\mathrm{c}}\right.$ is closer to zero and $\sigma_{\mathrm{r}}$ is further from zero). This does not appear to be a consequence of radial distance (since the orbit is geometrically symmetric) or the angle of the magnetic field: the distribution of $\theta_{\mathrm{BR}}$ (reflected to lie in the range of $0^{\circ}$ to $90^{\circ}$ ) is the same to within uncertainties, with a mean value of $\theta_{\mathrm{BR}}=26.8^{\circ} \pm 0.4^{\circ}$ in both cases. One key difference, however, is the proximity to the HCS, the effect of which is explored in the rest of this Letter.

One important consideration is whether the Taylor hypothesis remains valid as PSP gets closer to the Sun (Klein et al. 2015; Bourouaine \& Perez 2018, 2019, 2020). Figure 2 also contains the time series of the parameter $\epsilon=\delta v_{\text {rms }} / \sqrt{2} v_{\mathrm{sc}}$ calculated from 1-hour intervals, where $v_{\mathrm{sc}}$ is the magnitude of the solar wind velocity in the spacecraft frame. This is the same parameter as in the model of Bourouaine \& Perez (2019), in which perpendicular sampling is assumed so $v_{\mathrm{sc}} \sim v_{\mathrm{sc} \perp}$, and in which the Taylor hypothesis is valid for $\epsilon \ll 1$. The model also assumes Gaussian random sweeping and anisotropic turbulence $k_{\perp} \gg k_{\|}$, and it is valid when $\tan \left(\theta_{\mathrm{BV}}\right) \gtrsim \delta v_{\text {rms }} / v_{\mathrm{A}}$, where $\theta_{\mathrm{BV}}$ is the angle between $\mathbf{B}_{0}$ and the mean solar wind velocity in the spacecraft frame. Bourouaine \& Perez (2020) determined that within this model, frequency broadening caused by the breakdown of the Taylor hypothesis does not significantly modify the spectrum as long as $\epsilon \lesssim 0.5$, and as shown in Fig. 2, the data points satisfy this condition. Therefore, the differences in turbulence characteristics investigated in this Letter are likely not due to the differences in the validity of the Taylor hypothesis. A more detailed analysis of the Taylor hypothesis for these first PSP orbits is given in Perez et al. (2021).

\subsection{HCS proximity dependence}

In the outbound portion of Encounter 4, PSP spent significant time in the streamer belt wind near the HCS. The width of the streamer belt wind at these distances is not well known, so the dependence of the turbulence properties on the distance to the HCS was investigated. Figure 3 shows average values close to and far from the HCS, as a function of the cut value of the HCS angle used to define close and far, $\phi_{\text {cut }}$. For example, the first panel shows $\left\langle\delta B_{\text {rms }}\right\rangle_{\left|\phi_{\mathrm{HCS}}\right|<\phi_{\text {cut }}}$ as a function of $\phi_{\text {cut }}$ in blue and $\left\langle\delta B_{\text {rms }}\right\rangle_{\left|\phi_{\mathrm{HCS}}\right|>\phi_{\text {cut }}}$ as a function of $\phi_{\text {cut }}$ in red. Because the imbalance is so high, plots for the Elsasser ratio,

$r_{\mathrm{E}}=\frac{1+\sigma_{\mathrm{c}}}{1-\sigma_{\mathrm{c}}}$,

and Alfvén ratio,

$r_{\mathrm{A}}=\frac{1+\sigma_{\mathrm{r}}}{1-\sigma_{\mathrm{r}}}$

are also shown. All quantities show a difference at all cut angles, but the largest overall difference is between $\approx 3^{\circ}$ and $\approx 5^{\circ}$, so a value of $\phi_{\text {cut }}=4^{\circ}$ was used to define the width of the region near the HCS in which the turbulence properties are different.

Figure 4 shows the distributions of the turbulence properties over the encounter both near to $\left(\left|\phi_{\mathrm{HCS}}\right|<4^{\circ}\right)$ and far from $\left(\left|\phi_{\mathrm{HCS}}\right|>4^{\circ}\right)$ the HCS. A clear difference can be seen in each property: near the HCS, there are lower amplitudes, higher magnetic compressibility, a steeper spectrum, a lower level of imbalance, and a broader distribution of residual energy. The joint distributions of $\sigma_{\mathrm{c}}$ with $\sigma_{\mathrm{r}}$ and $r_{\mathrm{E}}$ with $r_{\mathrm{A}}$ are shown in Fig. 5. The data were constrained mathematically to lie within the regions

$\sigma_{\mathrm{c}}^{2}+\sigma_{\mathrm{r}}^{2} \leq 1$

and

$\left(\frac{r_{\mathrm{E}}-1}{r_{\mathrm{E}}+1}\right)^{2}+\left(\frac{r_{\mathrm{A}}-1}{r_{\mathrm{A}}+1}\right)^{2} \leq 1$, 

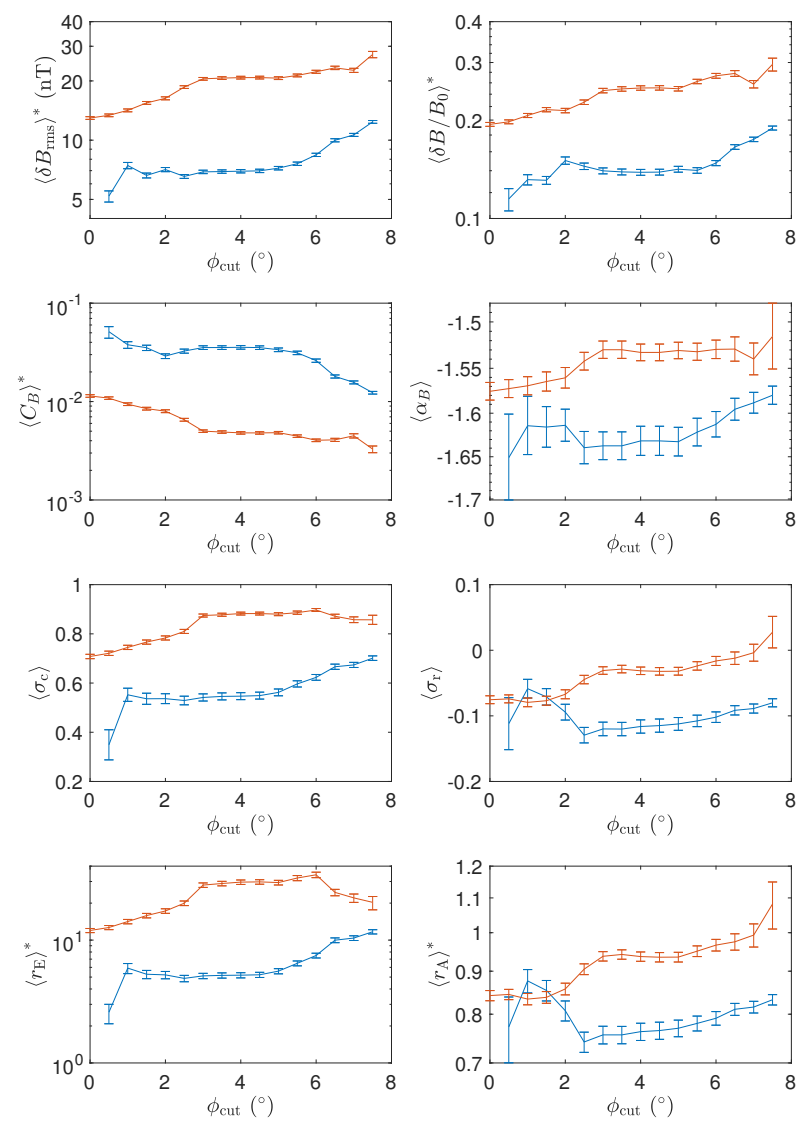

Fig. 3. Average turbulence properties for times close to (blue) and far from (red) the HCS as a function of the value $\phi_{\text {cut }}$ used to define close and far. Averages are arithmetic means except for quantities marked with *, which are geometric means. The error bars represent the standard error of the mean. The largest overall difference in the average values is at $\phi_{\text {cut }} \approx 4^{\circ}$.

respectively, marked as solid black lines. In general, it can be seen that in both cases the fluctuations are highly Alfvénic $\left(\sigma_{\mathrm{c}} \approx\right.$ $\left.1, \sigma_{\mathrm{r}} \approx 0, r_{\mathrm{E}} \gg 1, r_{\mathrm{A}} \approx 1\right)$, more so than in previous encounters (Chen et al. 2020; McManus et al. 2020; Parashar et al. 2020), but the near-HCS wind is less Alfvénic than the wind far from the HCS.

The averages for these different regions are given in Table 1. Aspects to take note of are that near the HCS, the wind is denser, slower, and cooler, with a higher plasma beta and mass flux, which is as expected for the streamer belt wind. The kinetic energy flux, however, is very similar, as has been seen previously across different wind types (Le Chat et al. 2012). The difference in turbulence properties is consistent with the differences between 'fast' and 'slow' wind seen further from the Sun (e.g. Tu \& Marsch 1995; Bruno \& Carbone 2013). Notably, the magnetic field spectral index in the streamer belt wind is close to $-5 / 3$, whereas it is closer to $-3 / 2$ far from the HCS. This is consistent with observed dependencies of the magnetic spectrum on the degree of Alfvénicity at 1 au (Podesta \& Borovsky 2010; Chen et al. 2013; Bowen et al. 2018) and in the previous PSP orbits (Chen et al. 2020). A similar difference in the spectral index and Alfvénicity was also seen when separating times of inverted and non-inverted magnetic field (Bourouaine et al. 2020). The imbalance, however, is larger than typically seen at $1 \mathrm{au}$, suggesting that the evolution towards a more balanced state seen in the open field wind (Chen et al. 2020) also occurs in the streamer belt wind.

L3, page 4 of 6
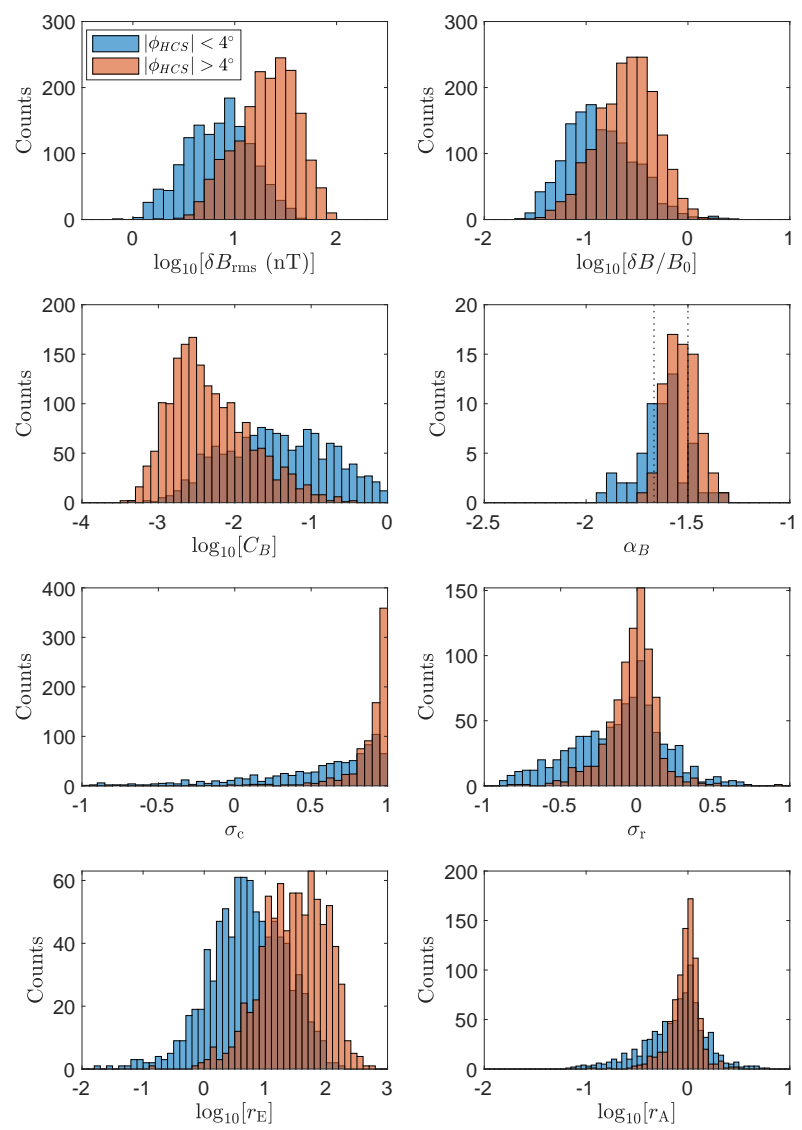

Fig. 4. Histograms of turbulence properties close to (blue) and far from (red) the HCS. A clear difference can be seen in all properties.
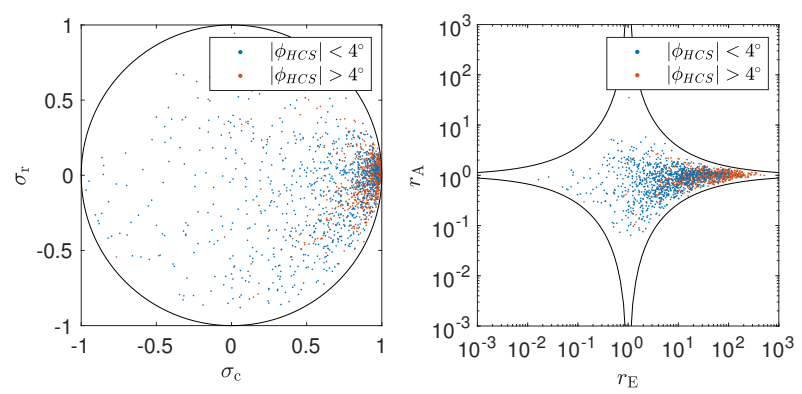

Fig. 5. Distributions of normalised cross helicity, $\sigma_{\mathrm{c}}$, normalised residual energy, $\sigma_{\mathrm{r}}$, Elsasser ratio, $r_{\mathrm{E}}$, and Alfvén ratio, $r_{\mathrm{A}}$, for times close to (blue) and far from (red) the HCS.

\subsection{Spectra}

Spectra of representative intervals (marked by the vertical dashed lines in Figs. 1 and 2) are shown in Fig. 6. The lower panel shows the local spectral index, in which some important differences can be seen. Firstly, the steeper magnetic field spectrum in the near-HCS interval can be seen throughout the MHD inertial range. But more significantly, the break between the ' $1 / f$ ' range and the MHD inertial range is at very different frequencies: $f_{\mathrm{b}} \approx 3 \times 10^{-4} \mathrm{~Hz}$ in the near-HCS wind and $f_{\mathrm{b}} \approx 4 \times 10^{-3} \mathrm{~Hz}$ far from the HCS. The origin of the $1 / f$ break is debated (Matthaeus \& Goldstein 1986; Velli et al. 1989; Verdini et al. 2012; Perez \& Chandran 2013; Wicks et al. 2013a,b; Chandran 2018; Matteini et al. 2018), but this difference is also seen between the 'fast' and 'slow' wind at 1 au (Bruno et al. 2019), where both breaks are about a decade lower in frequency, which is consistent with the radial evolution (Chen et al. 2020). 
Table 1. Solar wind and turbulence properties close to $\left(\left|\phi_{\mathrm{HCS}}\right|<4^{\circ}\right)$ and far from $\left(\left|\phi_{\mathrm{HCS}}\right|>4^{\circ}\right)$ the heliospheric current sheet (HCS).

\begin{tabular}{ccc}
\hline \hline Property & $\left|\phi_{\mathrm{HCS}}\right|<4^{\circ}$ & $\left|\phi_{\mathrm{HCS}}\right|>4^{\circ}$ \\
\hline$B(\mathrm{nT})$ & 56 & 88 \\
$n_{\mathrm{e}}\left(\mathrm{cm}^{-3}\right)$ & 510 & 390 \\
$v_{\mathrm{i}}\left(\mathrm{km} \mathrm{s}^{-1}\right)$ & 240 & 300 \\
$T_{\mathrm{i}}(\mathrm{eV})$ & 20 & 55 \\
$4 \pi \rho v_{\mathrm{r}} r^{2}\left(10^{-14} M_{\odot} \mathrm{yr}^{-1}\right)$ & 3.1 & 2.1 \\
$2 \pi \rho v_{\mathrm{r}}^{3} r^{2}\left(10^{19} \mathrm{~W}\right)$ & 6.0 & 6.0 \\
$\beta_{\mathrm{i}}^{*}$ & 1.2 & 0.68 \\
\hline$\delta B^{*}(\mathrm{nT})$ & 7.0 & 21 \\
$\left(\delta B / B_{0}\right)^{*}$ & 0.14 & 0.25 \\
$C_{B}^{*}$ & 0.036 & 0.0048 \\
$\alpha$ & -1.63 & -1.53 \\
$\sigma_{\mathrm{c}}$ & 0.55 & 0.88 \\
$\sigma_{\mathrm{r}}$ & -0.12 & -0.031 \\
$r_{\mathrm{E}}^{*}$ & 5.2 & 30 \\
$r_{\mathrm{A}}^{*}$ & 0.76 & 0.94 \\
$f_{\mathrm{b}}(\mathrm{Hz})$ & $3 \times 10^{-4}$ & $4 \times 10^{-3}$ \\
\hline & &
\end{tabular}

Notes. Quantities are arithmetic means, apart from those marked with ${ }^{*}$, which are geometric means.

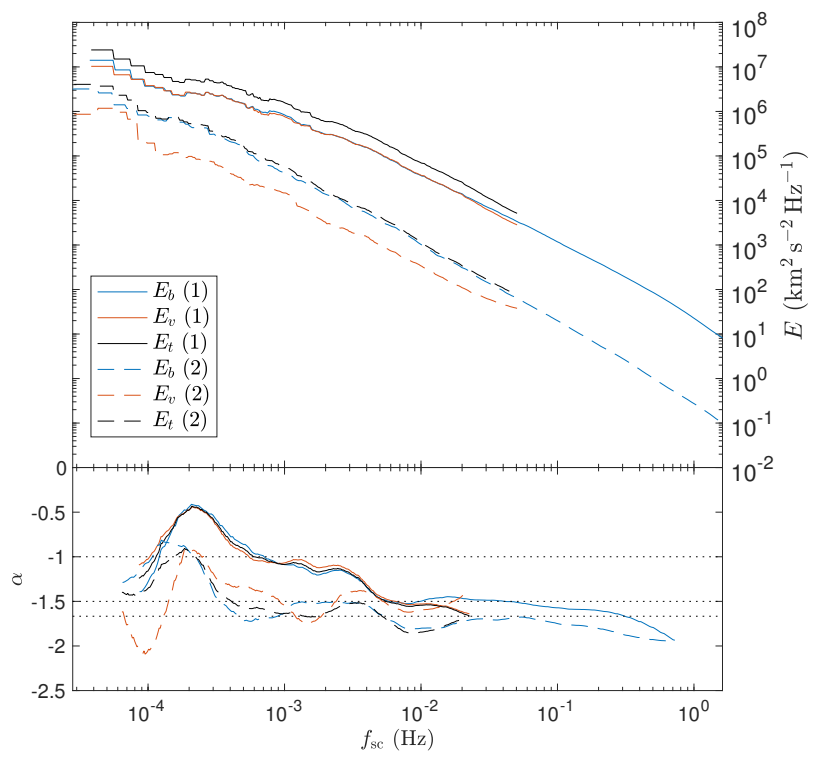

Fig. 6. Trace power spectra of magnetic, velocity, and total energy fluctuations $\left(E_{b}, E_{v}\right.$, and $\left.E_{t}\right)$ for intervals 1 and 2 (indicated in Figs. 1 and 2). Lower panel: local spectral index, $\alpha$, calculated over a sliding window of a factor of 5 .

\subsection{Energy flux}

Finally, the energy flux in the turbulent fluctuations was compared to the Chandran et al. (2011) reflection-driven turbulence solar wind model, following Chen et al. (2020). The Alfvénic turbulence enthalpy flux was calculated as

$F_{\mathrm{A}}=\rho|\delta \mathrm{b}|^{2}\left(\frac{3}{2} v_{\mathrm{r}}+v_{\mathrm{A}}\right)$,

where $v_{\mathrm{A}}=B_{0} / \sqrt{\mu_{0} \rho_{0}}$ is the Alfvén speed, and the solar wind bulk kinetic energy flux was calculated as

$F_{\mathrm{k}}=\frac{1}{2} \rho v_{\mathrm{r}}^{3}$.

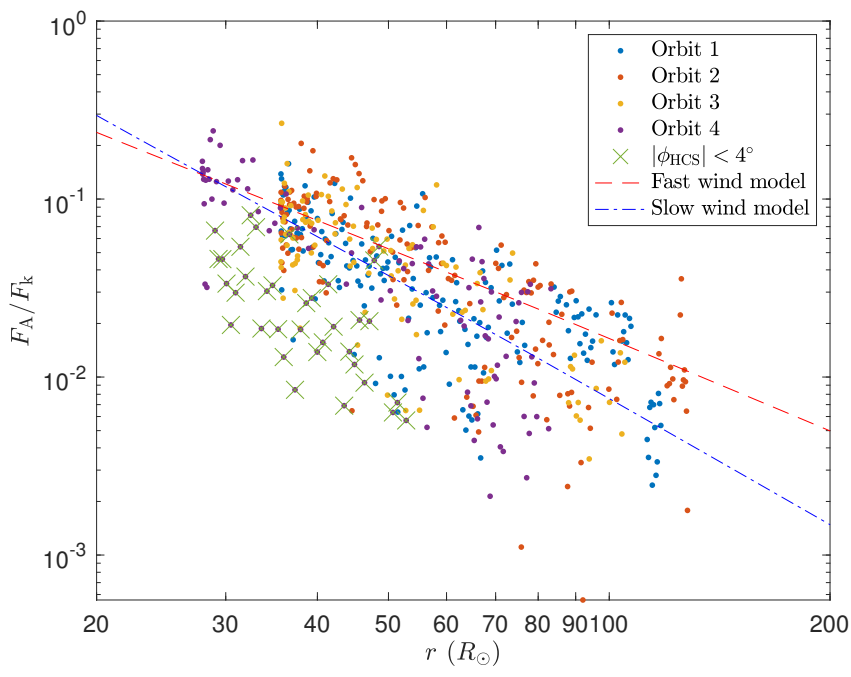

Fig. 7. Ratio of Alfvén wave energy flux, $F_{\mathrm{A}}$, to bulk solar wind kinetic energy flux, $F_{\mathrm{k}}$, for Orbits 1-4. Times during Orbit 4 which are close to the HCS $\left(\left|\phi_{\mathrm{HCS}}\right|<4^{\circ}\right)$ are marked with green crosses. The fast and slow wind solutions to the Chandran et al. (2011) model are shown with the red dashed and blue dash-dotted lines.

These quantities are similar to those plotted in Chandran et al. (2011) and Chen et al. (2020) except $\delta \mathbf{b}$ was used instead of $\delta \mathbf{z}^{+}$, which allows greater data coverage and is valid due to the high degree of Alfvénicity (Fig. 5). Figure 7 shows the ratio $F_{\mathrm{A}} / F_{\mathrm{k}}$, calculated over 3-hour intervals, as a function of solar distance over the first four orbits of PSP. The SPAN-I data were used for $v_{\mathrm{r}}$ for times when reliable fits could be made, and SPC data were used otherwise. Also shown are two solutions to the Chandran et al. (2011) model as described in Chen et al. (2020).

It can be seen that for Orbits 1-3, the data, on average, follow the model solutions (although with some degree of spread), consistent with the findings of Chen et al. (2020). For Orbit 4, however, there are a large number of points that fall well below the model, originating from times during the outbound portion of the encounter near the HCS when the turbulent amplitudes were lower. The energy flux ratio during times when the spacecraft was less than $4^{\circ}$ from the HCS during Encounter 4 is on average 3.0 times smaller than the slow wind model (whereas the average ratio to the model for the other data points over this distance range is 1.0). This suggests that the lower amplitude turbulence near the HCS does not contain sufficient energy for reflectiondriven Alfvénic turbulence models to provide an explanation for the acceleration of the streamer belt wind.

\section{Discussion}

In this Letter, it is shown that the turbulence properties in the near-Sun streamer belt wind, from $\approx 28 R_{\odot}$ to $\approx 54 R_{\odot}$, are significantly different than those in open field wind that has been measured for most of the previous PSP orbits. These differences include lower amplitudes, higher magnetic compressibility, a steeper magnetic spectrum $(-5 / 3$ rather than $-3 / 2)$, a lower degree of Alfvénicity, and a larger scale $1 / f$ break. The differences are similar to the traditional fast and slow wind differences reported at $1 \mathrm{au}$, suggesting the near-Sun streamer belt wind as the prototypical slow solar wind.

The differences in turbulence properties occur at an angle to the HCS of $\left|\phi_{\mathrm{HCS}}\right| \approx 4^{\circ}$, suggesting the total width of the streamer belt wind at these solar distances to $b e \approx 8^{\circ}$. This interpretation is consistent with other studies of the streamer belt 
wind during PSP Encounter 4, for example, Badman et al. (2021) found a reduced magnetic flux during this period that did not fit the general radial scalings seen in the rest of the PSP data. The inferred streamer belt wind width is also consistent with coronal images that show the streamer rays to have a width of around $10^{\circ}$ to $20^{\circ}$, which is likely a slight overestimate due to the line-of-sight integration (Rouillard et al. 2020; Poirier et al. 2020).

The Alfvénic turbulence energy flux measured on PSP's first three orbits is generally in line with that required for the reflection-driven solar wind model of Chandran et al. (2011); however, in the Encounter 4 streamer belt wind, it is several times lower than the model predictions. There are also occasional periods in the previous orbits where this is the case; they were identified by Chen et al. (2020) as the periods of quiet radial-field wind seen by Bale et al. (2019). This raises the possibility that these may be small patches of streamer belt wind; investigating this possibility would be an interesting topic for further study. The difference to the model predictions implies that purely reflection-driven Alfvénic turbulence solar wind models (e.g. Cranmer et al. 2007; Chandran et al. 2011) may not be able to account for the acceleration of the streamer belt wind, and additional processes are taking place. One possible process is additional turbulence driving by velocity shears (Usmanov et al 2018; Ruffolo et al. 2020). One thing to note about this possibility, however, is that it would be expected to produce both inward and outward Alfvén waves, resulting in $\sigma_{\mathrm{c}} \approx 0$ and $r_{\mathrm{E}} \approx 1$ if the shears dominate the energy input. In the streamer belt wind measured here, $\sigma_{\mathrm{c}}=0.55$ and $r_{\mathrm{E}}=5.2$, on average; this means that even though the imbalance is less than in the open field wind, there is still five times more energy in the outward waves compared to the inward ones. Therefore, this process may play a role but cannot be dominating the energy input overall. Another likely contribution to the generation of the streamer belt wind is reconnection in the near-Sun HCS (Lavraud et al. 2020) triggered by a tearing mode (Réville et al. 2020b). The chain of processes involved in the acceleration of the streamer belt wind, however, remains an open question. Future orbits of PSP closer to the Sun will allow more to be learnt about the nature of both plasma turbulence near the Sun and solar wind acceleration.

Acknowledgements. C.H.K.C. was supported by STFC Ernest Rutherford Fellowship ST/N003748/2 and STFC Consolidated Grant ST/T00018X/1. B.D.G.C. was supported in part by NASA grants NNN06AA01C, NNX17AI18G, and 80NSSC19K0829. L.D.W. was supported by STFC Consolidated Grant ST/S000364/1. J.C.P. was partially supported by NASA grants NNX16AH92G, 80NSSC19K0275 and NSF grant AGS-1752827. S.B. was supported by NASA grants NNX16AH92G, 80NSSC19K0275 and 80NSSC19K1390. K.G.K. was supported by NASA ECIP grant 80NSSC19K0912. S.D.B. was supported in part by the Leverhulme Trust Visiting Professorship programme. We thank the members of the FIELDS/SWEAP teams and PSP community for helpful discussions. PSP data is available at the SPDF (https://spdf.gsfc.nasa.gov).

\section{References}

Adhikari, L., Zank, G. P., \& Zhao, L. L. 2020, ApJ, 901, 102

Alazraki, G., \& Couturier, P. 1971, A\&A, 13, 380

Alexandrova, O., Chen, C. H. K., Sorriso-Valvo, L., Horbury, T. S., \& Bale, S. D. 2013, Space Sci. Rev., 178, 101

Arge, C. N., \& Pizzo, V. J. 2000, J. Geophys. Res., 105, 10465

Arge, C. N., Odstrcil, D., Pizzo, V. J., \& Mayer, L. R. 2003, AIP Conf. Proc., 679,190

Arge, C. N., Luhmann, J. G., Odstrcil, D., Schrijver, C. J., \& Li, Y. 2004, J. Atmos. Sol.-Terr. Phys., 66, 1295

Badman, S. T., Bale, S. D., Martínez Oliveros, J. C., et al. 2020, ApJS, 246, 23

Badman, S. T., Bale, S. D., Rouillard, A. P., et al. 2021, A\&A, 650, A18 (PSP SI)
Bale, S. D., Goetz, K., Harvey, P. R., et al. 2016, Space Sci. Rev., 204, 49 Bale, S. D., Badman, S. T., Bonnell, J. W., et al. 2019, Nature, 576, 237 Bandyopadhyay, R., Goldstein, M. L., Maruca, B. A., et al. 2020, ApJS, 246, 48 Barnes, A. 1992, Rev. Geophys., 30, 43

Belcher, J. W. 1971, ApJ, 168, 509

Bourouaine, S., \& Perez, J. C. 2018, ApJ, 858, L20

Bourouaine, S., \& Perez, J. C. 2019, ApJ, 879, L16

Bourouaine, S., \& Perez, J. C. 2020, ApJ, 893, L32

Bourouaine, S., Perez, J. C., Klein, K. G., et al. 2020, ApJ, 904, L30

Bowen, T. A., Mallet, A., Bonnell, J. W., \& Bale, S. D. 2018, ApJ, 865, 45

Bowen, T. A., Mallet, A., Bale, S. D., et al. 2020, Phys. Rev. Lett., 125, 025102

Bruno, R., \& Carbone, V. 2013, Liv. Rev. Sol. Phys., 10, 2

Bruno, R., Telloni, D., Sorriso-Valvo, L., et al. 2019, A\&A, 627, A96

Case, A. W., Kasper, J. C., Stevens, M. L., et al. 2020, ApJS, 246, 43

Chandran, B. D. G. 2018, J. Plasma Phys., 84, 905840106

Chandran, B. D. G., Dennis, T. J., Quataert, E., \& Bale, S. D. 2011, ApJ, 743 197

Chaston, C. C., Bonnell, J. W., Bale, S. D., et al. 2020, ApJS, 246, 71

Chen, C. H. K. 2016, J. Plasma Phys., 82, 535820602

Chen, C. H. K., Bale, S. D., Salem, C. S., \& Maruca, B. A. 2013, ApJ, 770, 125

Chen, C. H. K., Bale, S. D., Bonnell, J. W., et al. 2020, ApJS, 246, 53

Coleman, P. J. 1968, ApJ, 153, 371

Cranmer, S. R., van Ballegooijen, A. A., \& Edgar, R. J. 2007, ApJS, 171, 520

Cranmer, S. R., Asgari-Targhi, M., Miralles, M. P., et al. 2015, Phil. Trans. R. Soc. A, 373, 20140148

Cranmer, S. R., Gibson, S. E., \& Riley, P. 2017, Space Sci. Rev., 212, 1345

Duan, D., Bowen, T. A., Chen, C. H. K., et al. 2020, ApJS, 246, 55

Fox, N. J., Velli, M. C., Bale, S. D., et al. 2016, Space Sci. Rev., 204, 7

Hansteen, V. H., \& Velli, M. 2012, Space Sci. Rev., 172, 89

Heinemann, M., \& Olbert, S. 1980, J. Geophys. Res., 85, 1311

Hollweg, J. V. 2008, JApA, 29, 217

Kasper, J. C., Abiad, R., Austin, G., et al. 2016, Space Sci. Rev., 204, 131

Kiyani, K. H., Osman, K. T., \& Chapman, S. C. 2015, Phil. Trans. R. Soc. A, 373, 20140155

Klein, K. G., Perez, J. C., Verscharen, D., Mallet, A., \& Chandran, B. D. G. 2015 ApJ, 801, L18

Lavraud, B., Fargette, N., Réville, V., et al. 2020, ApJ, 894, L19

Le Chat, G., Issautier, K., \& Meyer-Vernet, N. 2012, Sol. Phys., 279, 197

Leer, E., Holzer, T. E., \& Fla, T. 1982, Space Sci. Rev., 33, 161

Livi, R., Larson, D. E., Kasper, J. C., et al. 2021, ApJS, submitted

Matteini, L., Stansby, D., Horbury, T. S., \& Chen, C. H. K. 2018, ApJ, 869, L32

Matthaeus, W. H., \& Goldstein, M. L. 1986, Phys. Rev. Lett., 57, 495

McManus, M. D., Bowen, T. A., Mallet, A., et al. 2020, ApJS, 246, 67

Meyrand, R., Squire, J., Schekochihin, A. A., \& Dorland, W. 2020, ArXiv eprints [arXiv:2009.02828]

Moncuquet, M., Meyer-Vernet, N., Issautier, K., et al. 2020, ApJS, 246, 44

Panasenco, O., Velli, M., D’Amicis, R., et al. 2020, ApJS, 246, 54

Parashar, T. N., Goldstein, M. L., Maruca, B. A., et al. 2020, ApJS, 246, 58

Parker, E. N. 1958, ApJ, 128, 664

Parker, E. N. 1965, Space Sci. Rev., 4, 666

Perez, J. C., \& Chandran, B. D. G. 2013, ApJ, 776, 124

Perez, J. C., Bourouaine, S., Chen, C. H. K., \& Raouafi, N. E. 2021, A\&A, 650, A22 (PSP SI)

Podesta, J. J., \& Borovsky, J. E. 2010, Phys. Plasmas, 17, 112905

Poirier, N., Kouloumvakos, A., Rouillard, A. P., et al. 2020, ApJS, 246, 60

Réville, V., Velli, M., Panasenco, O., et al. 2020a, ApJS, 246, 24

Réville, V., Velli, M., Rouillard, A. P., et al. 2020b, ApJ, 895, L20

Roberts, D. A., Goldstein, M. L., Matthaeus, W. H., \& Ghosh, S. 1992, J. Geophys. Res., 97, 17115

Rouillard, A. P., Kouloumvakos, A., Vourlidas, A., et al. 2020, ApJS, 246, 37

Ruffolo, D., Matthaeus, W. H., Chhiber, R., et al. 2020, ApJ, 902, 94

Shoda, M., Suzuki, T. K., Asgari-Targhi, M., \& Yokoyama, T. 2019, ApJ, 880, L2

Szabo, A., Larson, D., Whittlesey, P., et al. 2020, ApJS, 246, 47

Tu, C.-Y., \& Marsch, E. 1995, Space Sci. Rev., 73, 1

Usmanov, A. V., Matthaeus, W. H., Goldstein, M. L., \& Chhiber, R. 2018, ApJ, 865,25

van der Holst, B., Sokolov, I. V., Meng, X., et al. 2014, ApJ, 782, 81

Velli, M. 1993, A\&A, 270, 304

Velli, M., Grappin, R., \& Mangeney, A. 1989, Phys. Rev. Lett., 63, 1807

Verdini, A., Velli, M., Matthaeus, W. H., Oughton, S., \& Dmitruk, P. 2010, ApJ, 708, L116

Verdini, A., Grappin, R., Pinto, R., \& Velli, M. 2012, ApJ, 750, L33

Wicks, R. T., Mallet, A., Horbury, T. S., et al. 2013a, Phys. Rev. Lett., 110, 025003

Wicks, R. T., Roberts, D. A., Mallet, A., et al. 2013b, ApJ, 778, 177

Woodham, L. D., Horbury, T. S., Matteini, L., et al. 2021, A\&A, 650, L1 (PSP SI) 